\title{
Desarrollo de habilidades intelectuales en docentes de educación básica de Machala, Ecuador
}

\author{
EUDALDO ENRIQUE ESPINOZA FREIRE * \\ Universidad Técnica de Machala - Ecuador \\ MYRIAN LUZ RICALDI ECHEVARRIA ${ }^{*}$ \\ Universidad Femenina del Sagrado Corazón - Perú \\ Recibido el 23-01-18; primera evaluación el 26-06-19; \\ segunda evaluación el 10-07-19; tercera evaluación el \\ 07-08-19; aceptado el 20-08-19
}

\section{RESUMEN}

Las habilidades intelectuales generales constituyen las premisas para la formación de capacidades y competencias profesionales; sin embargo, se observan falencias en su implementación desde la enseñanza básica. Este estudio se realizó con el objetivo de caracterizar el proceso de desarrollo de estas habilidades en los alumnos de las escuelas básicas de Machala. La estrategia metodológica respondió al paradigma cuanti-cualitativo, sistematizada a través de los métodos de observación científica, fenomenológico, hermenéutico y estadístico; la información coleccionada a través de la aplicación de la guía de observación a 12 clases y la entrevista realizada a 40 docentes seleccionados aleatoriamente fueron resumidos a través de tablas y figuras estadísticas. Los hallazgos permitieron concluir que existen limitaciones en la intencionalidad del desarrollo de las habilidades intelectuales generales.

Palabras clave: Habilidades intelectuales, educación básica, observación.

\footnotetext{
* Doctor en Ciencias Pedagógicas por la Universidad de Oriente de la República de Cuba. Magíster en Gerencia de Educación Abierta y a Distancia por la Universidad Regional Autónoma de los Andes de Ecuador. Licenciado en Ciencias de la Educación por la Universidad Estatal de Guayaquil, especializado en Historia y Geografía. Participó en más de 60 eventos académicos. Fue ponente en más de 30 congresos científicos internacionales y realizó 39 publicaciones, de las cuales 33 son artículos científicos y 6 obras. Experiencia laboral de 23 años como docente. Correo electrónico: eespinoza@utmachala.edu.ec

** Magíster en Enseñanza de la Matemática por la Pontificia Universidad Católica del Perú. Licenciada en Educación por la Universidad Nacional Mayor de San Marcos. Participó en eventos internacionales de Educación Matemática. Sus líneas de investigación son aspectos socioculturales de la educación, pensamiento algebraico, pensamiento geométrico, y recursos para la enseñanza y el aprendizaje de la matemática. Correo electrónico: myrianluz@hotmail.com
} 


\section{Development of intellectual skills in teachers of basic education in Machala, Ecuador}

\section{Abstract}

The general intellectual abilities constitute the premises for the formation of professional abilities and competences; however, there are shortcomings in its implementation since basic education. This study was carried out with the objective of characterizing the development process of these skills in the students of the basic schools of Machala. The methodological strategy responded to the quantitative-qualitative paradigm, systematized through the methods of scientific observation, phenomenological, hermeneutic and statistical; the information collected through the application of the observation guide to 12 classes and the interview with 40 randomly selected teachers were summarized through tables and statistical figures. The findings allowed concluding that there are limitations in the intentionality of the development of general intellectual abilities.

Keywords: Intellectual skills, basic education, observation.

\section{Desenvolvimento de habilidades intelectuais em professores de educaçáo básica em Machala, Equador}

\section{RESUMO}

As habilidades intelectuais gerais constituem as premissas para a formação de habilidades e competências profissionais; No entanto, existem deficiências na sua implementação desde a educação básica. Este estudo foi realizado com o objetivo de caracterizar o processo de desenvolvimento dessas habilidades nos alunos das escolas básicas de Machala. A estratégia metodológica respondeu ao paradigma quantitativo-qualitativo, sistematizado através dos métodos de observação científica, fenomenológica, hermenêutica e estatística; as informaçôes coletadas através da aplicação do guia de observação para 12 aulas e a entrevista com 40 professores selecionados aleatoriamente foram resumidas através de tabelas e figuras estatísticos. Os achados permitiram concluir que existem limitaçóes na intencionalidade do desenvolvimento de habilidades intelectuais gerais.

Palavras-chave: habilidades intelectuais, educação básica, observação. 


\section{INTRODUCCIÓN}

En los discursos académicos, mucho se habla de la necesidad de egresados universitarios capaces de asumir eficientemente, desde su posición de profesionales, la dirección de los procesos vinculados con sus modos y esferas de actuación, así como formar en ellos competencias y habilidades para su desempeño integral (Luna, 2015).

Sin embargo, al respecto, Núñez et al. (2017) señalan que luego del estudio efectuado en una universidad privada de México con 27 alumnos, sobre el desarrollo de habilidades de pensamiento crítico, los resultados mostraron que el $50 \%$ aún se encuentra con limitaciones para dirigir procesos, integrar conocimientos, habilidades y valores, así como manifestación de debilidades en la aplicación práctica de lo aprendido y en su integración, incidiendo negativamente en el desarrollo profesional.

En tal sentido, se han realizado acciones y trazado estrategias desde las aulas universitarias para contribuir a la satisfacción de esta urgencia de la sociedad; sin embargo, no existe una suficiente mirada integradora de este fenómeno que encause no solamente su solución desde los procesos de formación profesional, sino y tan importante, desde los primeros años de la vida escolar, en donde se debe iniciar la formación de las habilidades necesarias para su ulterior desarrollo y florecimiento en los grados siguientes hasta el nivel superior; en este empeño, la formación de habilidades intelectuales generales es un eslabón crucial. Cañizares et al. (2014); Moreno y Tejeda (2017) consideran que son las que definen posteriormente competencias profesionales.

Es necesario señalar que, por lo general, en las aulas de la enseñanza básica se presta mayor importancia e interés al conocimiento que al resto de los componentes del proceso de enseńanza-aprendizaje; es por ello que, según Salazar et al. (2019), una de las grandes deficiencias que presentan los alumnos es el bajo nivel de desarrollo de las habilidades intelectuales. En este orden de ideas, Turner y Chávez (1989) expresaron que «en la práctica escolar ha existido la supremacía incondicional del volumen de conocimientos sobre el desarrollo de capacidades mentales» (p. 12). «Actitud contradictoria», pues cuando se habla de la calidad del aprendizaje, este no puede ser medido por el volumen de conocimiento que alcanza el estudiante, tan importante como ello es el desarrollo de las habilidades y valores que están estrechamente vinculados y constituyen el sistema de contenidos de la enseñanza, como lo manifiestan Morales et al. (2018). Para Agulló et al. (2017), las habilidades intelectuales generales están indisolublemente ligadas a la asimilación de los conocimientos como apoyo y sustento, a la vez que en ese mismo proceso de asimilación se desarrollan y perfeccionan. 
Diversos autores han abordado las habilidades intelectuales generales desde diferentes aristas, entre ellos: Turner y Chávez (1989); Castejón et al. (2004); Rodríguez et al. (2013); Kohler (2013); Cañizares et al. (2014); Griffin, McGaw y Care (2015); Care et al. (2016); Aznar y Laiton (2017); Frías et al. (2017); Montes y Torres (2017); Morales et al. (2018); Salazar y Tobón (2018) y Salazar et al. (2019).

En sus estudios, se refieren al desarrollo de habilidades intelectuales generales en los alumnos, a la necesaria preparación y capacitación de los maestros para su implementación y a la necesidad de establecer estrategias que permitan su formación desde las edades escolares. Coinciden en señalar que se observan insuficiencias en la preparación de los docentes, no existe una suficiente integración interdisciplinaria para su implementación y desenvolvimiento, cada disciplina desde sus currículos aborda las habilidades inherentes a sus materias; y no se explicitan en los currículos las metodologías para su desarrollo, solo de aquellas específicas de las asignaturas.

Existen falencias no ajenas al proceso de enseñanza-aprendizaje que se lleva a cabo en las aulas de la educación básica de las escuelas de la ciudad de Machala, donde se presentan insuficiencias en el aprendizaje de los alumnos, las que se manifiestan a través de calidad de la lectura, comprensión lectora, expresión escrita, expresión oral, solución de problemas, la argumentación de ideas, entre otras. Esta situación motivó el presente estudio con el propósito de caracterizar el proceso de desarrollo de las habilidades intelectuales generales que se lleva a cabo en la educación básica de Machala ${ }^{1}$.

\section{MarCo teórico}

\subsection{Breve acercamiento teórico en torno al desarrollo de las habilidades intelectuales en la enseñanza básica}

Muchas de las habilidades que precisan los profesionales de hoy en día para desenvolverse en un mundo globalizado y en constante cambio, gracias al desarrollo de la Ciencia y la Técnica, se comienzan a gestar desde los primeros años de la educación preescolar y básica, entre ellas, la observación, descripción y comparación (Lara, 2013; Montes y Torres 2017). De aquí surge la necesidad, por parte de los docentes, de su conocimiento teórico y puesta en práctica de estrategias didácticas que garanticen su formación y desarrollo.

\footnotetext{
Las escuelas de Educación Básica, objeto del presente estudio, están situadas en la ciudad de Machala, provincia El Oro, pertenecientes al distrito 07D02, Zona 7, del Ministerio de Educación de Ecuador.
} 
Existen variadas definiciones de "habilidad» aportadas por diversos autores. La consulta a fuentes bibliográficas especializadas permite exponer las siguientes:

Según González (1995), «las habilidades constituyen el dominio de operaciones (psíquicas y prácticas) que permiten una regulación racional de la actividad» (p. 117). Desde una perspectiva pedagógica, Rodríguez et al. (2017) las entienden como un concepto extraordinariamente complejo y amplio; es una capacidad adquirida por el hombre de utilizar racionalmente sus conocimientos y hábitos en el proceso de actividad teórica y práctica.

Para Álvarez de Zayas (1994), la habilidad expresa «la dimensión del contenido que muestra el comportamiento del hombre en una rama del saber propio de la cultura de la humanidad» (p. 71). Según este autor, desde el punto de vista psicológico, es el sistema de acciones y operaciones dominado por el sujeto que responde a un objetivo.

Curbeira et al. (2013) declaran:

como formación psicológica que tiene su fundamento en la estructura de la personalidad y, particularmente en la esfera de autorregulación ejecutora, en la que coexisten las unidades psíquicas: estado cognitivo, estado metacognitivo e instrumentación ejecutora, de la que es tributaria junto a otras manifestaciones de las ejecuciones: acciones, operaciones, hábitos y capacidades (p. 384).

Estos autores consideran que es la capacidad de realizar una operación compleja integral y representan una actividad creadora con una fuerte carga psicológica, la cual pone en juego un complejo sistema de acciones y operaciones que refleja el modo en que el sujeto realiza su actividad para lograr el objetivo propuesto.

Particularmente, Amorós (2009) considera que las habilidades intelectuales son aquellas que se necesitan en la realización de las actividades mentales. Por otra parte, Rosa Torres, citado en De Los Ángeles, A. y Ortiz (s.f), y Vargas y Gómez (2013), define las habilidades intelectuales como un "conjunto de aptitudes que optimizan el aprendizaje de nuevos conocimientos, complementándose con habilidades manuales, estéticas y demás propias del ser humano que, mostrando una actitud humanista, favorecen en gran medida el proceso de aprendizaje de nuevas habilidades» (p. 3).

Tomando en consideración las definiciones antes analizadas, se asume en este estudio como habilidades intelectuales aquellas que intervienen en la ejecución de actividades mentales para la transformación del objeto, lo que concuerda con Espinoza (2018), quien agrega que la adquisición del conocimiento es producto de los objetivos trazados en el proceso de aprendizaje 
y que favorecen la adquisición de nuevas habilidades. A lo antes mencionado, se complementa lo propuesto por Aquiles et al. (2013), quienes apuntan que las habilidades, desde una perspectiva didáctica, caracterizan las acciones que el alumno realiza para suplir la carencia de conocimiento frente a una situación desconocida y que engrosan el caudal de saberes contribuyendo a la formación intelectual de la personalidad.

Es importante señalar que resulta necesario que el alumno tenga la posibilidad de sistematizar las acciones y operaciones para lograr el desarrollo de la habilidad, lo cual está directamente relacionado con el nivel de asimilación de la actividad. Morales et al. (2018) sustentan que no es posible desarrollar una habilidad al margen del dominio de las acciones necesarias para alcanzar un objetivo propuesto. Siguiendo esta misma línea de análisis, Aznar y Laiton (2017), luego de haber hecho el estudio en un instituto politécnico de Bogotá con 21 estudiantes, en la asignatura de física I sobre Desarrollo de Habilidades Básicas de Pensamiento Crítico en el Contexto de la Enseñanza de la Física Universitaria, concluyen que «la formación de habilidades no se logra por la repetición de las acciones, sino por el efecto generalizador y de extrapolarización que se espera de ellos» (párr. 46). Es por ello que, durante la ejecución de la actividad de aprendizaje, se llevan a cabo la realización de diversos ejercicios, en los que pondrán en práctica las operaciones indicadas, propiciando que el educando se convierta en un sujeto activo, crítico, creativo y participativo, según proponen Frías et al. (2017); de ahí que sea importante que el docente en la programación de las actividades considere las características de los escolares en la selección de los métodos y los procedimientos.

En tal sentido, Aznar y Laiton (2017) precisan que el «desarrollo intelectual del alumno se logra en el proceso de aprehensión del contenido, donde se debe prever el desarrollo de las habilidades del pensamiento» (párr.18), teniendo en cuenta los objetivos, los contenidos, y la selección de los métodos. Agregan Aquiles et al. (2013) que al culminar las actividades resulta importante propiciar su control, lo que permite conocer el nivel de desarrollo de las acciones alcanzado por los escolares y que estos puedan analizar, comparar, valorar sus resultados, y con ello contribuir a desarrollar su pensamiento y habilidades intelectuales; garantizando así su desarrollo intelectual, integral y de la personalidad. Además, las habilidades intelectuales tienen una influencia considerable en la adquisición de los conocimientos y habilidades.

En correspondencia con estas ideas DGESPE (2012), reflexiona que es preciso que los docentes partan del criterio que las habilidades intelectuales se enseñan y aprenden; estas deberían ser un prerrequisito para el aprendizaje de habilidades básicas en el contexto académico. 


\subsection{Taxonomías de las habilidades intelectuales generales}

La escuela básica tiene, entre sus objetivos, la formación de habilidades que favorecen el desarrollo intelectual. Existen múltiples taxonomías, entre ellas, la propuesta por Hernández (2000), que contempla entre otras siete habilidades intelectuales generales: observar, describir, comparar, ejemplificar, utilizar modelos, clasificar e identificar, las que por el interés de este estudio se tuvieron en cuenta. A lo anterior, se suma lo propuesto por Rodríguez y Pérez (2017), quienes consideran que la observación de los objetos y los fenómenos del entorno ponen en contacto directo al alumno con el mundo que lo rodea, amplía sus conocimientos y desarrolla sus capacidades intelectuales. Para Isaza y Henao (2012), esta habilidad comienza a formarse en el niño desde edades bien tempranas y su desarrollo continúa en la enseñanza básica, a través de las actividades educativas.

Según Espinoza et al. (2018), es importante que el maestro tenga presente que el alumno no sabe observar por sí solo, que es necesario enseñarlo, el alumno debe aprender a observar para así asegurar la calidad de sus ideas. En primer lugar, es imprescindible conocer el estilo de aprendizaje y nivel de partida del escolar, cuál es el nivel alcanzado en el desarrollo de las acciones, de esta forma podrá hacer una adecuada selección del objeto y procedimientos para la realización de la observación.

Autores como Cańizares y Sarasa (2011) y Espinoza et al. (2018) declaran que un elemento a tener en consideración es despertar el interés del educando por el objeto de observación. Para ello, puede utilizarse charlas, mostrar láminas; además, es necesario orientar el objetivo y las tareas de la observación, exponer los procedimientos para la correcta observación, resumir los conocimientos obtenidos sobre el objeto y valorar los procedimientos utilizados, entre otras acciones. Este sistema de acciones abarca desde la introducción hasta las conclusiones, pasando por la ejecución de las operaciones.

El desarrollo de la habilidad de descripción tiene sus bases en la educación preescolar, como lo manifiesta Cárcel (2016). Su esencia radica en la verbalización de lo que el escolar percibe, por esta razón, contribuye grandemente al desarrollo del lenguaje. Los educandos pueden describir objetos reales y sus representaciones, láminas, hechos, procesos, en dependencia del objeto de estudio de que se trate. Esta habilidad guarda una estrecha relación con la observación, el niño describe lo que observa, solo después empieza a describir aquello que recuerda. Para facilitar la descripción el maestro, puede utilizar diferentes recursos: manipulación de objetos o un sistema de preguntas que permita al alumno orientarse en la tarea. 
La habilidad de comparación presupone la contraposición de las características de dos o más objetos para determinar sus semejanzas y diferencias. Tiene como base a la observación, por lo que se deduce que antes de enseñar a los alumnos a comparar es necesario haberlos enseñado a observar (Morales et al. 2018). La comparación de objetos, hechos y situaciones se realiza sobre la base de procesos de análisis, síntesis, abstracción y generalización.

La ejemplificación es una vía que garantiza el vínculo del contenido de la enseñanza con la vida del alumno y permite concretar en objetos de la realidad la generalización expresada en un hecho, concepto o ley, con lo que el aprendizaje se hace más asequible. Para Isaza y Henao (2012), es importante llegar a lograr que sean los escolares quienes ejemplifiquen los elementos del contenido de enseñanza, lo que facilita su consolidación y sistematización

La utilización de modelos se inicia a finales de la edad temprana cuando surgen en el niño las acciones intelectuales realizadas mentalmente sin pruebas externas. Para el desarrollo de esta habilidad, se utilizan simples de distintos tipos, instrumentos sencillos y medios materiales.

En la educación parvularia, los niños poseen modelos gráficos que constituyen una condición fundamental para la representación gráfica de los objetos; además, a esa edad inician en la construcción de objetos determinados que se les entregan en calidad de modelo (Calet et al. 2016). Al final de la edad preescolar, el nińo utiliza modelos esquemáticos, logro en el desarrollo del pensamiento infantil que permite establecer relaciones entre los objetos, hechos y fenómenos del medio, lo que puede considerarse como etapa propedéutica para el desarrollo de esta habilidad en los subsiguientes grados académicos.

Para Salazar et al. (2019), el empleo de modelos resulta sumamente importante para el aprendizaje, sobre todo en los primeros grados, debido a que, en gran medida, facilita la adquisición de conocimientos, sirve de base para realizar el control del trabajo y le permite al escolar valorar la calidad de sus acciones, determinar sus insuficiencias y organizar el trabajo para su corrección.

En opinión de Ramos et al. (2016), la habilidad de clasificación posibilita distribuir, organizar los objetos y fenómenos a partir de su pertenencia a una clase, género o grupo según la afinidad que existan entre ellos, teniendo en cuenta determinados criterios. Por su parte, la identificación permite establecer la identidad del objeto, hecho o fenómeno que se estudia partiendo de sus rasgos esenciales que lo distinguen de los demás. Las acciones de identificación están dirigidas hacia analizar y caracterizar el objeto, así como establecer relación del objeto con un hecho, concepto o ley de los conocidos. 
Para el desarrollo del proceso de enseñanza-aprendizaje, el maestro debe tener presente que entre las habilidades existen determinadas relaciones jerárquicas, como ya se ha apuntado anteriormente. A criterio de autores como Ackerman (1988); Sternberg y Lubart (1996); Cohen et al. (2000); Ambrose et al. (2003); Mumford (2003) y Ambrose (2005), la descripción permite evaluar la observación, sirve de base a la comparación y a la clasificación. Por otra parte, la utilización de modelos tiene como premisas la observación, la descripción y la comparación. La ejemplificación se basa en la identificación. La comparación tiene bases en la descripción y en los momentos iniciales, así como en la observación. La clasificación tiene como premisas la comparación, la descripción, la observación y la identificación. Hay que tener presente estos elementos en su desarrollo, respetar la subordinación lógica de estas, cuidar el orden a seguir y dónde comenzar, pues no respetarse esto determina las insuficiencias en su desarrollo y, por consiguiente, en la calidad de la personalidad en formación.

\section{Metodología}

La estrategia metodológica respondió al paradigma cuanti-cualitativo, transcurrió esencialmente a través del empleo de los métodos de investigación: observación científica, fenomenológica, hermenéutica y estadística, fundamentada, desde la posición de Padua (2018), para la recolección de datos teóricos y empíricos, y que posibilitó la triangulación con el fin de caracterizar el proceso de desarrollo de las habilidades intelectuales generales en los alumnos de la enseñanza básica en la ciudad de Machala. Para la observación científica directa al proceso de enseñanza-aprendizaje, se consideró lo propuesto por Rositas-Martínez (2017), quien sostiene que la observación a la práctica educativa ofrece objetividad en el proceso investigativo, situación requerida para determinar las regularidades en función a la formación y fomento de estas habilidades y el desarrollo alcanzado por los alumnos. La fenomenología aseguró el conocimiento de las creencias, criterios y opiniones de los maestros sobre el fenómeno objeto de estudio. Por su parte, la hermenéutica facilitó el estudio y análisis crítico de la literatura especializada para la valoración del estado del arte. Así como para la revisión de los documentos normativos y planes de clases, se siguió lo propuesto por Ambrosio (2018). El empleo del método estadístico se dio siguiendo lo propuesto por Badii et al. (2017), quienes mencionan que una vez recolectada la información, se hace necesario procesarla; posterior a ello, analizar, interpretar y comprender la problemática estudiada, situación que fue necesaria en la planificación, recolección, procesamiento y análisis de la información coleccionada a través de la aplicación de la guía de 
observación a 12 clases y la entrevista realizada a los 40 docentes seleccionados aleatoriamente. En cuanto a la entrevista, se optó por ser estandarizada, con la finalidad de no influir en las respuestas. Una vez explicado el cuestionario, se pidió que las respuestas las ubiquen por escrito y así evitar influir en sus contestaciones, lo que concuerda con las ideas de Corvetta (2003). Para la confección de los instrumentos aplicados (guía de observación y entrevista), se adoptó como directriz metodológica la validada por Hernández (2000), siendo este un instrumento confiable, además por estar acorde a los requerimientos metodológicos de la presente investigación.

La guía de observación fue elaborada teniendo en cuenta los siguientes objetivos:

- Determinar la intencionalidad del desarrollo de las habilidades intelectuales en los alumnos mediante la ejecución de las clases y revisión de la planificación.

- Determinar el nivel de desarrollo de estas habilidades a través de las manifestaciones de los alumnos durante la clase.

- Comprobar la puesta en práctica de estrategias docentes dirigidas al desarrollo de las habilidades intelectuales generales.

La entrevista estandarizada aplicada a los 40 maestros tuvo como objetivos los siguientes:

- Conocer el grado de preparación de los docentes para asumir el desarrollo de habilidades intelectuales generales y cómo desarrollan las acciones para lograr tal propósito.

- Obtener información sobre la contribución de sus disciplinas en el desarrollo de habilidades intelectuales de carácter general.

- Conocer las opiniones y criterios sobre el proceso de desarrollo de las habilidades intelectuales generales.

La entrevista siguió el siguiente orden de interrogantes:

- ¿Qué habilidades intelectuales debe desarrollar en sus alumnos, según el grado que cursan?

- ¿Elaboras estrategias didácticas intencionadas al fomento y desarrollo de las habilidades intelectuales?

- ¿Qué habilidades intelectuales observadas tienen desarrolladas tus educandos? 


\section{Resultados y Discusiones}

A partir de los resultados obtenidos, a través de la observación a las clases y entrevista a los docentes, se analizaron aspectos concernientes al desarrollo de las habilidades intelectuales en las escuelas de la enseñanza básica de la ciudad de Machala.

\subsection{Resultados de la observación a 12 clases de las escuelas de la educación básica de Machala}

\section{A. Análisis Cuantitativo}

Se pudo apreciar que en el 100\% (12) de las clases observadas, no existió intencionalidad en el desarrollo de las habilidades intelectuales generales de los alumnos, limitando su accionar a las habilidades inherentes a la asignatura, las que evidentemente contribuyen de alguna forma a este desarrollo intelectual, pero no de manera planificada y estructurada lógicamente. Esta situación es visible también en la planificación de las clases donde no se explicitan las acciones para el desarrollo de las habilidades intelectuales, dejándose a la espontaneidad del docente.

Mediante la observación al proceso de enseñanza-aprendizaje, se pudo determinar, a través de las manifestaciones y participación de los alumnos durante la clase, que en general el nivel de desarrollo de estas habilidades es bajo, el cual se resume en la siguiente tabla.

Tabla. 1. Observación. Desarrollo de habilidades intelectuales.

Educación Básica. Machala. Período Académico 2017-2018

\begin{tabular}{lcccccccc}
\hline \multirow{2}{*}{\multicolumn{1}{c}{ Habilidad }} & Cant. & \multicolumn{7}{c}{ Desarrollo } \\
\cline { 3 - 9 } & Alumnos & No & $\%$ & Inadecuado & $\%$ & Adecuado & $\%$ \\
\hline Observación & 250 & 75 & 30,4 & 137 & 54,4 & 38 & 15,2 \\
Descripción & 250 & 83 & 33,2 & 137 & 54,8 & 30 & 12 \\
Comparación & 250 & 91 & 36,4 & 148 & 59,2 & 11 & 4,4 \\
Ejemplificación & 250 & 96 & 38,4 & 146 & 58,4 & 8 & 3,2 \\
Utilización de modelos & 250 & 215 & 86 & 30 & 12 & 5 & 2 \\
Clasificación & 250 & 101 & 40,4 & 136 & 54,4 & 13 & 5,2 \\
Identificación & 250 & 98 & 39,2 & 144 & 57,6 & 8 & 3,2 \\
\hline
\end{tabular}

Fuente: Registro de observaciones. Elaborado por autores. 
Los datos de la tabla permiten apreciar que el $86 \%$ (215) de los alumnos no mostraron dominio de la habilidad de utilización de modelos, solo el $2 \%$ (5) reveló un adecuado desarrollo; en el resto de las habilidades, más del 30\% de los estudiantes, en cada caso, no mostró a través de sus acciones el debido desarrollo; únicamente el 15,2\% (38) sabe observar adecuadamente, describir el 12\% (30) y clasificar 5,2\% (13). El 4,4\% (11) evidenció dominar la comparación y el 3,2\% (8) sabe ejemplificar e identificar.

Asimismo, en el 100\% (12) de las clases observadas, se comprobó que no existen estrategias diseñadas para el desarrollo de las habilidades intelectuales generales. Por otra parte, en los análisis efectuados, al concluir las visitas al proceso de enseñanza-aprendizaje, se determinó que el 83,3\% (10) de las clases se planifican y ejecutan centrando la actividad en el maestro, lo que limitó la participación de los estudiantes; además, predominó el carácter reproductivo y estuvieron dirigidas a los conocimientos, desatendiendo los demás componentes del sistema de contenidos (habilidades y valores).

\section{B. Análisis Cualitativo}

Las principales dificultades, observadas en la tabla 1, sobre las manifestaciones del dominio de las habilidades de los alumnos estuvieron centradas en el cumplimiento de los objetivos trazados y de los pasos lógicos ejecutados con este fin; lo que difiere de lo propuesto por Morales et al. (2018), quienes sostienen que para comparar es necesario seguir el siguiente orden de operaciones: «determinar los objetos de comparación, determinar las líneas de comparación, determinar semejanzas y diferencias entre los objetos para cada línea de comparación y elaborar las conclusiones» (p. 53). Estas observaciones, además, permitieron determinar si existía o no tal desarrollo y si este era adecuado o inadecuado

Las insuficiencias apuntadas anteriormente inciden en la calidad de la enseñanza, traducidas en un pobre dominio de conocimientos y habilidades necesarias que sirven de base para el desarrollo de un aprendizaje exitoso. En virtud de ello, Ramírez et al. (2017) estiman que la valoración de estas falencias han permitido llegar al criterio de que un número considerable de maestros, que laboran en la enseñanza básica, presentan dificultades para efectuar acciones que favorezcan el desarrollo de las habilidades intelectuales de carácter general expresadas en los objetivos de este nivel de enseñanza, las que podrían ser resueltas a través de las diferentes estrategias didáctico-metodológicas que garanticen tanto la preparación metodológica de los maestros como el desarrollo de esas habilidades. De acuerdo con esto, Valdés et al. (2001), en sus estudios, concluyeron que «la efectividad en la formación y perfeccionamiento 
de las habilidades intelectuales se logra mediante estrategias donde se estructuren correctamente los pasos a seguir para que la acción devenga en habilidad, si se hace de forma sistemática, continua y consciente» (p. 293).

Los resultados obtenidos corresponden a lo señalado por Espinoza et al. (2018), en su trabajo sobre Reflexiones sobre una estrategia para enseñar geografía en la Educación Básica de Ecuador, quienes precisan que las actividades docente-educativas que se planifican son dirigidas por el maestro sin otorgar el papel protagónico a los alumnos y que fundamentalmente poseen un carácter reproductivo.

De igual manera, los docentes en sus clases prestan, de manera general, mucha más atención a los objetivos de sus programas de estudio y la evaluación está dirigida esencialmente a los conocimientos, desatendiendo los demás componentes del contenido que se plantean para los diferentes grados. La valoración de estas insuficiencias permitió llegar al criterio de que un número considerable de los docentes presentan dificultades para ejecutar estrategias que favorezcan el desarrollo de las habilidades intelectuales generales.

\subsection{Resultados de la entrevista a 40 docentes de las escuelas de la educación básica de Machala}

Al cuestionar a los docentes sobre qué habilidades intelectuales debe desarrollar en sus alumnos, según el grado que cursan, las respuestas se resumen en la figura 1 .

Figura 1. Habilidades intelectuales. Docentes de educación básica de la ciudad de Machala. Período académico 2017-2018

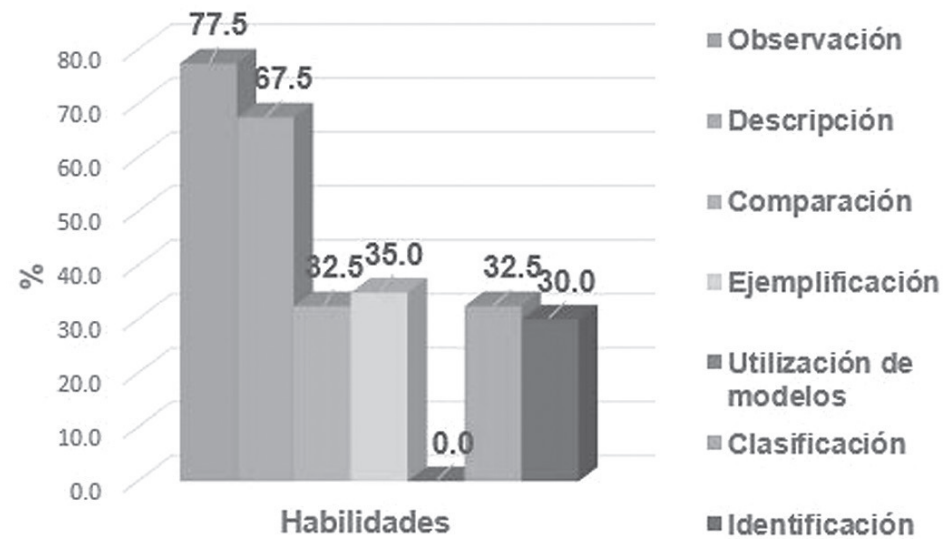

Fuente: Entrevista a docentes. Elaborado por autores. 


\section{A. Análisis Cuantitativo}

El 77,5\% (31) de los maestros entrevistados reconocieron la observación como una de las habilidades intelectuales generales a desarrollar en los alumnos en la educación básica; el 67,5\% (27), la descripción; el 32,5\% (13), la comparación y la clasificación; y el 35\% (14), la ejemplificación. La habilidad menos reconocida fue la identificación con $30 \%$ (12) y la utilización de modelos no fue reconocida por ninguno de los docentes.

\section{B. Análisis Cualitativo}

En la entrevista, se pudo determinar que algunos maestros tienen insuficiente conocimiento sobre las habilidades intelectuales que corresponden al grado en que trabajan; presentan un mejor dominio de las habilidades particulares de las asignaturas de Historia, Matemática y Lengua Materna, lo que se corresponde con los resultados de las averiguaciones realizadas por Hernández (2000) y Montes y Torres (2017), quienes encontraron que la inmensa mayoría de los docentes encuestados hacen referencia solamente a las habilidades específicas de los programas de las asignaturas Lengua Materna y Matemática.

Las indagaciones sobre la elaboración de estrategias didácticas intencionadas al fomento y desarrollo de las habilidades intelectuales propiciaron la información que se sintetiza en la figura 2.

Figura 2. Estrategias didácticas para el desarrollo de habilidades intelectuales. Docentes de educación básica de la ciudad de Machala. Período académico 2017-2018

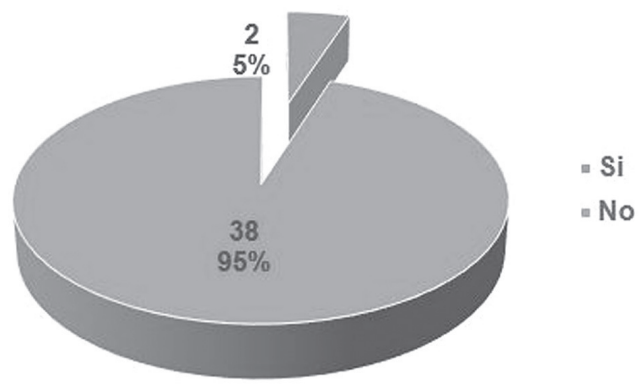

Fuente: Entrevista a docentes. Elaborado por autores.

\section{A. Análisis Cuantitativo}

En la figura, se puede observar que solo 2 (5\%) maestros declaran que existen estrategias diseñadas para el desarrollo de las habilidades intelectuales generales, mientras que los 38 (95\%) restantes no opinan de esa manera. 


\section{B. Análisis Cualitativo}

En este sentido, según las consideraciones de Ortiz y Marińo (2004) y Ramírez et al. (2017), el proceso de formación y desarrollo de las habilidades es complejo y requiere de un adecuado tratamiento multidisciplinar durante los diferentes grados, integrado en un sistema de acciones, que conforman las estrategias didácticas en las que se deben establecer etapas de planificación, orientación, ejecución, control y análisis.

Los resultados en las investigaciones de Kohler (2013) demuestran que el «rendimiento académico se asocia de forma significativa y positiva, con las habilidades intelectuales y con el uso de estrategias de aprendizaje de los alumnos» (p. 283); es decir, «el rendimiento académico de los estudiantes requiere de un conjunto de habilidades cognitivas y sociales que les permitan responder con efectividad a las exigencias propias del nivel educativo» (p. 283).

Por otro lado, existe correspondencia entre los resultados obtenidos en el presente estudio y las indagaciones realizadas por Hernández (2000) y Aquiles et al. (2013), donde ningún maestro expuso los requerimientos que deben ser tenidos en cuenta en las estrategias para el desarrollo de las habilidades. Además, ninguno se refirió a las acciones de orientación ni a las de control.

Con respecto a la pregunta planteada a los maestros sobre las habilidades intelectuales; es decir, opiniones desarrolladas sobre sus alumnos, se obtuvo la información que se brinda en la figura 3.

Figura 3. Habilidades intelectuales desarrolladas en los alumnos. Docentes de educación básica de la ciudad de Machala. Período académico 2017-2018

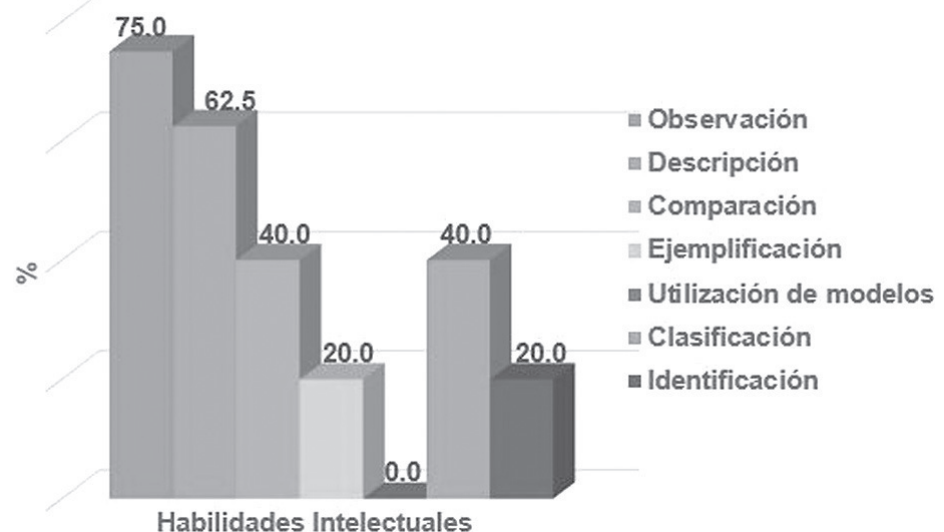

Fuente: Entrevista a docentes. Elaborado por autores. 


\section{A. Análisis Cuantitativo}

El 75\% (30) de los maestros opinan que sus alumnos tienen desarrollada la habilidad de observación; el 62,5\% (25), la de descripción; el 40\% (16) es del criterio que sus estudiantes saben comparar y clasificar; el 20\% (8) estima que pueden ejemplificar e identificar; y ningún profesor consideró la utilización de modelos.

\section{B. Análisis Cualitativo}

El análisis de esta información permite aseverar que existen dificultades en la formación de las habilidades que ocupan un nivel superior en la jerarquía del sistema de habilidades intelectuales, como es el caso de utilización de modelos, situación similar a la encontrada por Ramírez et al. (2017). Ninguno de los maestros consideró que sus educandos han desarrollado esa habilidad, lo que se corresponde con el dominio del conocimiento que posee estos (ver figura 1). En tal sentido, Hernández (2000) expresa que, en muchas ocasiones, los errores de los escolares se producen por la incompetencia del docente para realizar procedimientos lógicos de subordinación jerárquica y, en otros casos, por el desconocimiento.

Además, en esta entrevista, los docentes aportaron otras informaciones: El 87,5\% (35) consideran que deben ser capacitados metodológicamente para poder desarrollar de manera integral las habilidades intelectuales generales en sus alumnos, lo que coincide con los resultados de las investigaciones de Hernández (2000) que atribuyen las causas fundamentales de las dificultades en la implementación de estas habilidades a la insuficiente preparación de pregrado y a que no existe preocupación, en los diferentes niveles de dirección, por el desarrollo de estas habilidades.

\section{Conclusiones}

Los resultados de las averiguaciones realizadas, a nivel epistemológico y de campo, permiten concluir que el proceso de desarrollo de las habilidades intelectuales generales en los centros educacionales de la enseñanza básica de la ciudad de Machala presenta las siguientes falencias:

- Existe coincidencia en los referentes analizados que las habilidades intelectuales inciden positivamente en el proceso formativo desde la universidad y en el posterior desempeño de los egresados.

- Que las habilidades se forman a lo largo de toda la vida, por lo tanto, en la universidad, recae esa responsabilidad en los docentes, para lo cual es preciso considerar las particularices de los individuos. 
- No existe intencionalidad en el desarrollo de las habilidades intelectuales y está limitado a las habilidades inherentes a las asignaturas del currículo de estudio.

- No existen estrategias didácticas diseñadas para su implementación ni un adecuado tratamiento multidisciplinar, a través de los diferentes grados.

- Las clases se planifican y ejecutan centrado en la actividad del maestro, lo que limita la participación de los estudiantes; estas poseen carácter reproductivo y están dirigidas a los conocimientos, desatendiendo los demás componentes del sistema de contenidos (habilidades y valores).

- Existen dificultades en la formación de las habilidades intelectuales que ocupan un nivel superior en la jerarquía del sistema, como es el caso de utilización de modelos.

- Los docentes estiman no estar totalmente capacitados metodológicamente para desarrollar, de manera integral, las habilidades intelectuales generales en sus alumnos.

Como consecuencia de estas insuficiencias, el nivel de desarrollo de las habilidades intelectuales generales en los alumnos es bajo.

\section{REFERENCIAS BIBLIOGRÁFICAS}

Ackerman, P. L. (1988). Determinantes de las diferencias individuales durante la adquisición de habilidades: habilidades cognitivas y procesamiento de información. Revista de Psicología Experimental General, 117, 288-318.

Agulló, G., Meroño, C. y Bueno, Á. (2017). Educación emocional en la universidad: propuesta de actividades para el desarrollo de habilidades sociales y personales. Vivat Academia, (139), 1-17. https://doi.org/10.15178/ va.2017.139.1-17

Álvarez de Zayas, C. (1994). Epistemología. La Habana: Universidad de La Habana.

Ambrose, D. (2005). Creatividad en la enseñanza: conocimientos esenciales, habilidades y disposiciones. Creatividad a través de dominios: Caras de la musa, 281-298.

Ambrose, D., Cohen, L. M. y Tannenbaum, A. J. (eds.) (2003). Creative intelligence: Toward theoretic integration. Cresskill, NJ: Hampton Press.

Ambrosio, R. (2018). La socioformación: un enfoque de cambio educativo. Revista Iberoamericana de Educación, 76(1), 57-82. https://doi.org/10.35362/ rie7612955 
Amorós, E. (2009). Comportamiento organizacional. En busca del desarrollo de ventajas competitivas. Biblioteca virtual de Derecho, Universidad Católica, Santo Toribio de Mogrovejo, Escuela de Economía y Ciencias Sociales. Lambayeque, Perú.

Aquiles, J., Valdez, C. y Salellas, J. (2013). La adquisición de habilidades de razonamiento clínico en estudiantes de la carrera de Medicina. Revista Humanidades Médicas, 13(1), 72-87.

Aznar, I. y Laiton, I. (2017). Desarrollo de Habilidades Básicas de Pensamiento Crítico en el Contexto de la Enseńanza de la Física Universitaria. Revista Formación Universitaria, 10(1), 71-78. https://doi.org/10.4067/ S0718-50062017000100008

Badii, M. H., Castillo, J. y Cortez, K. (2017). Papel de la estadística en la investigación científica. Innovaciones de Negocios, 4(7).

Calet, N., Flores, M., Jiménez, G. y Defior, S. (2016). Habilidades fonológicas suprasegmentales y desarrollo lector en nińos de Educación Primaria. Revista Anales de PsicologialAnnals of Psychology, 32(1), 72-79. https://doi. org/10.6018/analesps.32.1.216221

Cañizares, O. y Sarasa, N. (2011). Posicionamiento didáctico para el desarrollo de habilidades intelectuales en ciencias básicas biomédicas. Edumecentro, 3(3), 22-27.

Cañizares, O., Sarasa, N., Villar, M., Pérez, A. y Morales, X. (2014). Aprender a observar desde el inicio de los estudios médicos es una necesidad formativa. Universidad de Ciencias Médicas. Sancti Spiritus. Gaceta Médica Espirituana, 16(2), 13-21.

Cárcel, F. (2016). Desarrollo de habilidades mediante el aprendizaje autónomo. Revista Tres Ciencias, empresa, investigación y pensamiento crítico, 5(3), 54-62. https://doi.org/10.17993/3cemp.2016.050327.52-60

Care, E., Scoular, C. y Griffin, P. (2016). Evaluación de la resolución colaborativa de problemas en entornos educativos. Revista Aplicada en Educación, 29(4). https://doi.org/10.1080/08957347.2016.1209204

Castejón, J., Prieto, M., Pérez, A. y Gilar, P. (2004). El rol del conocimiento y de las habilidades intelectuales generales en la adquisición del aprendizaje complejo. Universidad de Alicante. Psicothema, 16(4), 600-605. Recuperado de http://www.psicothema.com.

Cohen, L. M., Ambrose, D. y Powell, W. N. (2000). Conceptual foundations and theoretical lenses for the diversity of giftedness and talent. En K.A. Heller, F.J. Monks, R.J. Sternberg y R.F. Subotnik (eds.), International Handbook of Giftedness and Talent (pp. 331-344). Oxford: Elsevier. 
Corvetta, P. (2003). Metodología y técnicas de investigación social. Madrid: McGraw-Hill.

Curbeira, D., Bravo, M. y Bravo, G. (2013). La formación inicial de habilidades profesionales del ingeniero industrial desde el contexto de la matemática. Revista Ciencia y Sociedad, 38(2), 377-403. https://doi.org/10.22206/ cys.2013.v38i2.pp377-403

De Los Ángeles, A. y Ortiz, A. (s.f). Diagnóstico de 27 habilidades intelectuales de los alumnos de primer y tercer semestres de la Escuela Normal Superior Veracruzana Dr. Manuel Suárez Trujillo. X Congreso Nacional de Investigación Educativa, 1-17. Recuperado de http://www.comie.org.mx/congreso/ memoriaelectronica/v10/pdf/area_tematica_01/ponencias/0637-F.pdf

DGESPE (2012). Perfil de Egreso. Dirección General de Educación Superior para Profesionales de la Educación. México. Recuperado de http://www.gobmx/ funcionpublica.gob.mx.

Espinoza, E. (2018). Los medios como componentes del proceso de enseñanzaaprendizaje en estudios sociales en Machala, Ecuador. Maestro y Sociedad, 15(3), 359-373.

Espinoza, E., Calvas, M. y Chuquirima, S. (2018). Reflexiones sobre una estrategia para enseñar geografía en la Educación Básica de Ecuador. Maestro y Sociedad, 109-120.

Frías, M., Haro, Y. y Artiles, I. (2017). Las habilidades cognitivas en el profesional de la Información desde la perspectiva de proyectos y asociaciones internacionales. Investigación bibliotecológica, 31(71), 201-218. https://doi. org/10.22201/iibi.0187358xp.2017.71.57816

González, M. V. (1995). Psicología para educadores. Ciudad de La Habana: Pueblo y Educación.

Griffin, P., McGaw, B. y Care, E. (2015). Evaluación y enseñanza de las habilidades del siglo XXI: métodos y enfoque. Segunda ed. Dordrecht, Países Bajos: Springer.

Hernández, C. M. (2000). Propuesta de un diseño curricular para el desarrollo de habilidades intelectuales (Tesis de maestría). Instituto Superior Pedagógico Enrique José Varona Facultad de Ciencias de la Educación. La Habana, Cuba.

Isaza, L. y Henao, G. (2012). Influencia del clima sociofamiliar y estilos de interacción parental sobre el desarrollo de habilidades sociales en niños y nińas. Revista Persona, (15) 253-271. https://doi.org/10.26439/persona2012.n015.138

Kohler, J. (2013). Relación entre habilidades intelectuales, estrategias de aprendizaje y rendimiento académico en estudiantes de psicología del $1^{\circ}$ al $4^{\circ}$ 
ciclo de una universidad particular. Revista Liberabit, 19(2), 277-288. Recuperado de http://www.scielo.org.pe/pdf/liber/v19n2/a13v19n2.pdf

Lara, A. (2013). Desarrollo de habilidades de pensamiento y creatividad como potenciadores de aprendizaje. Revista Unimar, 30(1).

Luna, C. (2015). El futuro del aprendizaje. ¿Qué tipo de aprendizaje se necesita en el siglo XXI? Investigación y Prospectiva en Educación. Documentos de Trabajo ERF, No. 14. París: Unesco.

Montes, M. y Torres, J. (2017). Las competencias socio-afectivas docentes y la formación para la práctica educativa del desarrollo personal y para la convivencia, en el marco de la educación inclusiva. Revista de Educación Inclusiva, 8(3), 271-284.

Morales, L., García, O., Torres, A. y Lebrija, A. (2018). Habilidades cognitivas a través de la estrategia de aprendizaje cooperativo y perfeccionamiento epistemológico en Matemática de estudiantes de primer año de universidad. Formación Universitaria, 11(2), 45-56. https://doi.org/10.4067/ S0718-50062018000200045

Moreno, W. y Tejeda, E. (2017). Estrategia didáctica para desarrollar el pensamiento crítico. Revista Iberoamericana sobre Calidad, Eficacia y Cambio en Educación, 15(2), 53-73 https://doi.org/10.15366/reice2017.15.2.003

Mumford, M. D. (2003). ¿Dónde hemos estado, a dónde vamos? En balance en la investigación de la creatividad. Creativity Research Journal, 15(2-3), 107-120. https://doi.org/10.1207/S15326934CRJ152\&3_01

Núñez, S., Ávila, J. y Olivares, S. (2017). El desarrollo del pensamiento crítico en estudiantes universitarios por medio del Aprendizaje Basado en Problemas. Revista Iberoamericana de Educación Superior, 8(23), 84-103. https://doi. org/10.22201/iisue.20072872e.2017.23.3012

Ortiz, E. y Mariño, M. (2004). Estrategias educativas y didácticas en la educación superior. Pedagogía Universitaria, 9(5), 25 -26.

Padua, J. (2018). Técnicas de investigación aplicadas a las ciencias sociales. Ciudad de México, México: Fondo de Cultura Económica.

Ramírez, P., Graus, E., Castillo, D. y Rodríguez, F. (2017). El diagnóstico de la función orientadora en la formación inicial del profesional de la educación. Boletin Redipe, 6(3) 147-171.

Ramos, H., Villena, R. y Fernández, F. (2016). Los juguetes como medio de desarrollo del niño de la primera infancia. Atenas, 1(33), 110-120.

Rodríguez, A. y Pérez, A. (2017). Métodos científicos de indagación y de construcción del conocimiento. Revista EAN, (82), 179-200. https://doi. org/10.21158/01208160.n82.2017.1647 
Rodríguez, A., Valdez, C., García, C. y Casas, L. (2013). Habilidades de razonamiento clínico en estudiantes de la carrera de medicina. Revista de Humanidades Médicas, 13(2), 433-456.

Rodríguez, F., Cano, R. y Almaguer, S. (2017). Las competencias de dirección en educación. Una aproximación a su definición, estudio e interrelaciones en un contexto moderno, globalizado y complejo. Opuntia Brava, 9(1), 280-290.

Rositas-Martínez, J. (2017). Los tamaños de las muestras en encuestas de las ciencias sociales y su repercusión en la generación del conocimiento. Innovaciones de Negocios, 11(22), 235-268.

Salazar, E. y Tobón, S. (2018). Análisis documental del proceso de formación docente acorde con la sociedad del conocimiento. Espacios, 39(45), 17.

Salazar, O., Llaja, C., Macassi, L. y Rojas, O. (2019). Desarrollo de habilidades investigativas en estudiantes de enfermería: Nuevos retos en el contexto formativo. Investigación Valdizana, 13(2), 107-112. https://doi. org/10.33554/riv.13.2.236

Sternberg, R. J. y Lubart, T. I. (1996). Investing in creativity. American Psychologist, 51(7), 677-678. https://doi.org/10.1037//0003-066X.51.7.677

Turner, L. y Chávez, J. (1989). Se aprende a aprender. Ciudad de La Habana, Cuba: Pueblo y Educación.

Valdés, C., Álvarez, N., Valls, M., Valle, T. y Fajardo, B. (2001). Estrategia para desarrollar habilidades intelectuales en la asignatura BioquímicaI en estudiantes de medicina. Revista Cubana Educación Médica Superior, 15(2), 293-300.

Vargas, L., Gómez, M. y Gómez, R. (2013). Desarrollo de habilidades cognitivas y tecnológicas con aprendizaje móvil. Revista de Investigación Educativa del Tecnológico de Monterrey, 3(6), 30-39. 